Journal of Applied AnALysis

Vol. 9, No. 1 (2003), pp. 139-147

\title{
ON A CERTAIN GENERALIZATION OF THE KRASNOSEL'SKII THEOREM
}

\author{
M. GALEWSKI
}

Received July 3, 2001 and, in revised form, March 26, 2002

\begin{abstract}
We provide a generalization of a well known Krasnosel'skii theorem on continuity of the Nemytskii operator for functions taking values in separable Banach spaces. We follow the results obtained in [6] for the finite dimensional case.
\end{abstract}

\section{Introduction}

In [8] a short and elementary proof of the following Krasnosel'skii theorem on continuity of the Nemytskii operator is given

Theorem $1.1([9])$. Let $\Omega \subset \mathbb{R}^{k}$ be a Lebesgue measurable set. Let $f$ : $\Omega \times \mathbb{R}^{n} \rightarrow \mathbb{R}^{m}$ be a Caratheodory function, i.e. a function measurable with respect to the first variable and continuous with respect to the second one. Let $p_{1}, p_{2} \in[1, \infty]$. Assume there exist a constant $a>0$ and a function $b \in L^{p_{2}}[\Omega ; \mathbb{R}]$ such that for a.e. $t \in \Omega$ and for all $x \in \mathbb{R}^{n}$ we have

$$
\|f(t, x)\|_{\mathbb{R}^{m}} \leq a\|x\|_{\mathbb{R}^{n}}^{p_{1} / p_{2}}+b(t) .
$$

Then the Nemytskii operator $F$

$$
F: L^{p_{1}}\left[\Omega ; \mathbb{R}^{n}\right] \ni x(\cdot) \mapsto f(\cdot, x(\cdot)) \in L^{p_{2}}\left[\Omega ; \mathbb{R}^{m}\right]
$$

2000 Mathematics Subject Classification. 46T20, 35R99.

Key words and phrases. Nemytskii operator, continuity, Krasnosel'skii theorem.

ISSN 1425-6908（C) Heldermann Verlag. 
is continuous.

The same result is contained in [1]. Both proofs are based on Lemma IV.9, [4], although that in [8] is an indirect one.

The above theorem and some other results treat only the finite dimensional case. While in applications to abstract Hamiltonian systems [5], continuity of the following operator $F$ has to be shown

$$
F: L^{p_{1}}\left[0, T ; X_{1}\right] \ni x(\cdot) \mapsto f(\cdot, x(\cdot)) \in L^{p_{2}}\left[0, T ; X_{2}\right]
$$

Here $X_{1}, X_{2}$ are separable Hilbert spaces. The integral we understand in the sense of Bochner [7], [14]. Since the continuity of the above operator is not proved in [5] we provide an abstract version of Theorem 1.1. This theorem may also be used for example when applying a dual variational method, derived for ODE in [13], to showing the existence of solutions to a certain type of evolution equations.

Let $(M, \mathcal{B}, m)$ be a measure space and let $m(M)<\infty$. Let $X_{i}$ be separable Banach spaces for $i=1,2$. Let $p_{1}, p_{2} \in[1, \infty)$. We consider the spaces of $\mathcal{B}$-measurable mappings $x: M \rightarrow X_{i}$ such that

$$
\int_{M}\|x(s)\|_{X_{i}}^{p_{i}} d m<\infty
$$

$i=1,2$ which we denote by $L^{p_{i}}\left[M ; X_{i}\right]$. It is well known that for separable spaces all the definitions of measurability are equivalent. The abstract version of the Krasnosel'skii theorem reads:

Theorem 1.2. Let $f: M \times X_{1} \rightarrow X_{2}$ be a Caratheodory function, i.e. a function measurable with respect to the first variable for all $x \in X_{1}$ and continuous with respect to the second one for a.e. $t \in M$. Assume there exist a constant $a>0$ and a function $b \in L^{p_{2}}[M ; \mathbb{R}]$ such that for a.e. $t \in M$ and for all $x \in X_{1}$ we have

$$
\|f(t, x)\|_{X_{2}} \leq a\|x\|_{X_{1}}^{p_{1} / p_{2}}+b(t)
$$

Then the Nemytskii operator $F$

$$
F: L^{p_{1}}\left[M ; X_{1}\right] \ni x(\cdot) \mapsto f(\cdot, x(\cdot)) \in L^{p_{2}}\left[M ; X_{2}\right]
$$

is continuous.

The condition given in the above theorem is only a sufficient one. In case of finite dimensional spaces necessary conditions for continuity of the Nemytskii operator also exist, compare [2].

The paper is organized as follows. In Section 2 we prove the Krasnosel'skii theorem and generalize Theorem 1.2 [8], to the case which we consider. In Section 3 we provide an application. 


\section{Proof of the Krasnosel'skii theorem and some generalization}

In order to prove Theorem 1.2 we shall need the following

Lemma $2.1([7])$. Let $\left\{x_{n}\right\}$ be a sequence of elements of $L^{p}[M ; X]$ strongly converging to $x$ in $L^{p}[M ; X]$, where $1 \leq p<\infty, X$ is a Banach space. Then there exists a subsequence $\left\{x_{n_{k}}\right\} \in L^{p}[M ; X]$ such that $\lim _{k \rightarrow \infty} x_{n_{k}}(t)=$ $x(t)$ for a.e. $t \in M$. Moreover there exists a function $g \in L^{p}[M ; \mathbb{R}]$ satisfying the following condition

$$
\left\|x_{n_{k}}(t)\right\|_{X} \leq g(t) \text { for a.e. } t \in M .
$$

The above lemma as it is stated here is not to be found in [7] but it follows directly from the proof of Theorem 3.7.7 [7].

Now we are in the position to prove the Krasnosel'skii theorem.

Proof. Suppose that $x_{n} \rightarrow x$ in $L^{p_{1}}\left[M ; X_{1}\right]$ and that $f\left(\cdot, x_{n}(\cdot)\right)$ does not converge in $L^{p_{2}}\left[M ; X_{2}\right]$ to $f(\cdot, x(\cdot))$, i.e. there exists an $\varepsilon>0$, such that for all $N$ there exist an $n_{k} \geq N$ for which

$$
\int_{M}\left\|f(t, x(t))-f\left(t, x_{n_{k}}(t)\right)\right\|_{X_{2}}^{p_{2}} d m>\varepsilon .
$$

By (2.1) we may find a subsequence $\left\{x_{n_{k}}\right\}$ of $\left\{x_{n_{k}}\right\}$ - still denoted by $\left\{x_{n_{k}}\right\}$ - such that $x_{n_{k}}(t) \rightarrow x(t)$ a.e. on $M$ and for which there exists a function $g \in L^{p_{1}}[M ; \mathbb{R}]$ satisfying the condition

$$
\left\|x_{n_{k}}(t)\right\|_{X_{1}} \leq g(t) \text { for a.e. } t \in M .
$$

By continuity of $f$ with respect to the second variable we obtain that $f\left(t, x_{n_{k}}(t)\right) \rightarrow f(t, x(t))$ for $k \rightarrow \infty$ for a.e. $t \in M$. Using several times inequality $|a+b|^{p} \leq 2^{p-1}\left(|a|^{p}+|b|^{p}\right), p \geq 1$, we obtain for a.e. $t \in M$ by (1.1) that

$$
\begin{aligned}
& \left\|f(t, x(t))-f\left(t, x_{n_{k}}(t)\right)\right\|_{X_{2}}^{p_{2}} \leq 2^{p_{2}-1}\left(\|f(t, x(t))\|_{X_{2}}^{p_{2}}+\left\|f\left(t, x_{n_{k}}(t)\right)\right\|_{X_{2}}^{p_{2}}\right) \\
& \leq 2^{p_{2}-1}\left(\left(a\|x(t)\|_{X_{1}}^{p_{1} / p_{2}}+b(t)\right)^{p_{2}}+\left(a\left\|x_{n_{k}}(t)\right\|_{X_{1}}^{p_{1} / p_{2}}+b(t)\right)^{p_{2}}\right) \\
& \leq 2^{2 p_{2}-2}\left(a^{p_{2}}\left(\|x(t)\|_{X_{1}}^{p_{1}}+\left\|x_{n_{k}}(t)\right\|_{X_{1}}^{p_{1}}\right)+2 b^{p_{2}}(t)\right) \\
& \leq 2^{2 p_{2}-2}\left(a^{p_{2}}\left(\|x(t)\|_{X_{1}}^{p_{1}}+g^{p_{1}}(t)\right)+2 b^{p_{2}}(t) .\right)
\end{aligned}
$$

Of course the function

$$
M \ni t \mapsto 2^{2 p_{2}-2}\left(a^{p_{2}}\left(\|x(t)\|_{X_{1}}^{p_{1}}+g^{p_{1}}(t)\right)+2 b^{p_{2}}(t)\right) \in \mathbb{R}
$$


belongs to $L^{1}[M ; \mathbb{R}]$. Now by Lebesgue dominated convergence theorem we obtain

$$
\lim _{k \rightarrow \infty} \int_{M}\left\|f(t, x(t))-f\left(t, x_{n_{k}}(t)\right)\right\|_{X_{2}}^{p_{2}} d m=0
$$

which contradicts $(2.2)$.

The above theorem may be generalized as in [8]. Let $S_{i}$ for $i=1,2, \ldots, n$ denote the spaces of measurable functions on $M$ taking values in $X_{i}$, where $X_{i}$ is a separable Banach space . Let $L_{i}$, for $i=1,2, \ldots, n$, be such a subspace of $S_{i}$ that every sequence in $L_{i}$ contains a subsequence converging a.e. (cf. Lemma 2.1). Moreover assume that $f: M \times X_{1} \times \ldots \times X_{n}$ is a Caratheodory function. Define $L$ to be a subspace of the product $L_{1} \times \ldots \times L_{n}$.

Theorem 2.2. If for any sequence $\left\{\left(x_{k}^{1}, x_{k}^{2}, \ldots, x_{k}^{n}\right)\right\}_{k=1}^{\infty}$ in $L$ there exist a subsequence $\left\{\left(x_{k_{i}}^{1}, x_{k_{i}}^{2}, \ldots, x_{k_{i}}^{n}\right)\right\}_{i=1}^{\infty}$ and a function $h \in L^{p}[M ; X]$, where $1 \leq p<\infty$, such that

$$
\left\|f\left(t, x_{k_{i}}^{1}, x_{k_{i}}^{2}, \ldots, x_{k_{i}}^{n}\right)\right\|_{X} \leq\|h(t)\|_{X} \text {, for } i \in N \text { and a.e. } t \in M,
$$

than the Nemytskii operator

$$
F: L \ni\left(x^{1}, x^{2}, \ldots, x^{n}\right) \longmapsto f\left(\cdot, x^{1}(\cdot), x^{2}(\cdot), \ldots, x^{n}(\cdot)\right) \in L^{p}[M ; X],
$$

is well defined and continuous on $L$.

Proof. The indirect proof repeats that of the Krasnosel'skii Theorem 1.2 using the assumed existence of an almost everywhere convergent subsequence.

\section{Application}

In variational and optimal control problems an essential role is played by the theorem on the existence and continuity of the Fréchet derivative of integral functional, see [11], [12] and references therein. Since the case of functionals appearing in abstract O.D.E is considered in [5], we provide an example of such a functional which is of use when variational methods are applied in investigating evolution equations. As an easy corollary of Theorem 1.2 the continuity of the Fréchet derivative of the action functional form [5] may be proved.

Let $\Omega \subset \mathbb{R}^{k}$ be an open set with a smooth boundary $\partial \Omega$. Let $k_{i}$ be a nonnegative integer and consider for $i=1,2, \ldots, n$ the following spaces

$$
W^{1, k_{i}}(0, T)=\left\{x \in L^{p_{i}}\left[0, T ; H^{k_{i}+2}(\Omega)\right], \dot{x} \in L^{p_{i}}\left[0, T ; H^{k_{i}}(\Omega)\right]\right\},
$$


where the derivative we understand in the weak of the space $H^{k_{i}}(\Omega)$ and $H^{k_{i}}(\Omega)$ is the standard Sobolev space, [3], [6]. The norm on $W^{1, p_{i}}(0, T)$ reads

$$
\|x\|=\sqrt[p_{i}]{\int_{0}^{T}\|x(t)\|_{H^{k_{i}+2}(\Omega)}^{p_{i}} d t+\int_{0}^{T}\|\dot{x}(t)\|_{H^{k_{i}(\Omega)}}^{p_{i}} d t}
$$

Here $p_{i} \geq 2$ for $i=1,2, \ldots, n$. The function $x \in W^{1, k_{i}}(0, T)$ is continuous as a function $x:[0, T] \rightarrow H^{k_{i}+1}(\Omega)$ and its maximum norm satisfies the following estimation with the constant $C_{i}=C\left(T, k_{i}, \Omega\right)$

$$
\max _{0 \leq t \leq T}\|x(t)\|_{H^{k_{i}+1}(\Omega)} \leq C_{i}\left(\int_{0}^{T}\|x(t)\|_{H^{k_{i}+2}(\Omega)}^{2} d t+\int_{0}^{T}\|\dot{x}(t)\|_{H^{k_{i}(\Omega)}}^{2} d t\right)
$$

Put $W=W^{1, p_{1}} \times W^{1, p_{2}} \times \ldots \times W^{1, p_{n}}$. Let $q_{i}$ and $p_{i}$ be conjugate exponents for $i=1, \ldots, n$. Put $X=\prod_{i=1}^{n} H^{k_{i}+2}(\Omega) \times \prod_{i=1}^{n} H^{k_{i}}(\Omega)$ and denote its elements by $(x, \dot{x})$.

In what follows we denote

$$
\mathbb{R}_{0}^{+}=[0,+\infty), \quad \mathbb{R}_{+}=(0,+\infty) .
$$

Theorem 3.1. Let $g:[0, T] \times X \rightarrow \mathbb{R}$ be a Lebesgue measurable function in $t$ for every $(x, \dot{x}) \in X$ and continuous and continuously differentiable with respect to the last $2 n$ variables for a.e. $t \in[0, T]$. Assume there exist a continuous function $a \in C\left(\mathbb{R}^{n}, \mathbb{R}_{+}\right)$, a function $b \in L^{1}\left[0, T, \mathbb{R}_{0}^{+}\right]$, functions $c_{i} \in L^{q_{i}}\left[0, T ; \mathbb{R}_{0}^{+}\right], i=1, \ldots, n$, such that the following conditions are satisfied for $a\left(\left\|x_{1}\right\|, . .,\left\|x_{n}\right\|\right)=a\left(\left\|x_{1}\right\|_{H^{k_{1}+1}(\Omega)}, . .,\left\|x_{n}\right\|_{H^{k_{n}+1}(\Omega)}\right)$ for $i=1,2, \ldots, n$

$$
\begin{aligned}
& g(t, x, \dot{x}) \leq a\left(\left\|x_{1}\right\|, . .,\left\|x_{n}\right\|\right)\left(b(t)+\sum_{i=1}^{n}\|\dot{x}\|_{H^{k_{i}(\Omega)}}^{p_{i}}\right) \\
& \left\|\nabla_{x_{i}} g(t, x, \dot{x})\right\|_{H^{k_{i}(\Omega)}} \\
& \leq a\left(\left\|x_{1}\right\|, . .,\left\|x_{n}\right\|\right)\left(b(t)+\sum_{j=1}^{n}\left\|\dot{x}_{j}\right\|_{H^{k_{j}}(\Omega)}^{p_{j}}\right) \\
& \left\|\nabla_{\dot{x}_{i}} g(t, x, \dot{x})\right\|_{H^{k_{i}(\Omega)}} \\
& \leq a\left(\left\|x_{1}\right\|, . .,\left\|x_{n}\right\|\right)\left(c_{i}(t)+\sum_{j \neq i}^{n}\left\|\dot{x}_{j}\right\|_{H^{k_{j}(\Omega)}}^{p_{j} / q_{i}}+\left\|\dot{x}_{i}\right\|_{H^{k_{i}(\Omega)}}^{p^{k_{i}-1}}\right) .
\end{aligned}
$$

Then the functional

$$
F: W \ni x \rightarrow \int_{0}^{T} g(t, x, \dot{x}) d t \in \mathbb{R}
$$


is continuously Fréchet differentiable. Moreover the Fréchet differential $W \ni h \rightarrow F^{\prime}(x) h \in \mathbb{R}$ is given by the formula

$$
\begin{aligned}
F^{\prime}(x) h= & \sum_{i=1}^{n} \int_{0}^{T}\left\langle\nabla_{x_{i}} g(t, x(t), \dot{x}(t)), h_{i}(t)\right\rangle_{H^{k_{i}}(\Omega)} \\
& +\left\langle\nabla_{\dot{x}_{i}} g(t, x(t), \dot{x}(t)), \dot{h}_{i}(t)\right\rangle_{H^{k_{i}(\Omega)}} d t .
\end{aligned}
$$

Proof. Let us first observe that by (3.1) it follows that functional $F$ is well defined. We shall first show that for every direction $h \in W$ there exists a directional derivative. Let $\lambda \in \mathbb{R}$ and let $G(\lambda)=g(t, x(t)+\lambda h(t), \dot{x}(t)+$ $\lambda \dot{h}(t)) . G: \mathbb{R} \rightarrow \mathbb{R}$ is a differentiable function. For $\lambda \in[-1,1]$ we obtain

$$
\begin{aligned}
\frac{d}{d \lambda} G(\lambda)= & \sum_{i=1}^{n}\left\langle\nabla_{x_{i}} g(t, x(t)+\lambda h(t), \dot{x}(t)+\lambda \dot{h}(t)), h_{i}(t)\right\rangle_{H^{k_{i}(\Omega)}} \\
& +\sum_{i=1}^{n}\left\langle\nabla_{\dot{x}_{i}} g(t, x(t)+\lambda h(t), \dot{x}(t)+\lambda \dot{h}(t)), \dot{h}_{i}(t)\right\rangle_{H^{k_{i}(\Omega)}} .
\end{aligned}
$$

Using (3.2), (3.3) we get

$$
\begin{aligned}
& \left|\frac{d}{d \lambda} G(\lambda)\right| \leq a\left(\left\|x_{1}+\lambda h_{1}(t)\right\|, \ldots,\left\|x_{n}+\lambda h_{n}(t)\right\|\right) \\
& \times\left(\sum_{i=1}^{n}\left(b(t)+\sum_{j=1}^{n}\left\|\dot{x}_{j}(t)+\lambda \dot{h}_{j}(t)\right\|_{H^{k_{j}(\Omega)}}^{p_{j}}\right)\left\|h_{i}(t)\right\|_{H^{k_{i}(\Omega)}}\right. \\
& \left.+\sum_{i=1}^{n}\left(c_{i}(t)+\sum_{j \neq i}^{n}\left\|\dot{x}_{j}+\lambda \dot{h}_{j}(t)\right\|_{H^{k_{j}(\Omega)}}^{\frac{p_{j}}{q_{i}}}+\left\|\dot{x}_{i}\right\|_{H^{k_{i}(\Omega)}}^{p_{i}-1}\right)\left\|\dot{h}_{i}(t)\right\|_{H^{k_{i}(\Omega)}}\right)
\end{aligned}
$$

By continuity of $a: \mathbb{R}^{n} \rightarrow \mathbb{R}_{+}$and continuity of $x, h:[0, T] \rightarrow H^{k_{i}+1}(\Omega)$ we obtain that

$$
\begin{aligned}
& \max _{(t, \lambda) \in[0, T] \times[-1,1]} a\left(\left\|x_{1}(t)+\lambda h_{1}(t)\right\|_{H^{k_{1}+1}(\Omega)}, \ldots,\left\|x_{n}(t)+\lambda h_{n}(t)\right\|_{H^{k_{n}+1}(\Omega)}\right) \\
& =c .
\end{aligned}
$$

Application of inequalities $|a+b|^{p} \leq 2^{p-1}\left(|a|^{p}+|b|^{p}\right)$ and $|\lambda| \leq 1$ yields

$$
\begin{aligned}
\left|\frac{d}{d \lambda} G(\lambda)\right| \leq & c\left\{\sum _ { i = 1 } ^ { n } \left(b(t)+\sum_{j=1}^{n} 2^{p_{i-1}}\left(\left\|\dot{x}_{j}(t)\right\|_{H^{k_{j}}(\Omega)}^{p_{j}}\right.\right.\right. \\
& \left.\left.+\left\|\dot{h}_{j}(t)\right\|_{H^{k_{j}(\Omega)}}^{p_{j}}\right)\left\|h_{i}(t)\right\|_{H^{k_{i}}(\Omega)}\right)
\end{aligned}
$$




$$
\begin{aligned}
& +\sum_{i=1}^{n}\left(c_{i}(t)+\sum_{j \neq i}^{n} 2^{p_{j} / q_{i}-1}\left(\left\|\dot{x}_{j}\right\|_{H^{k_{j}(\Omega)}}^{p_{j} / q_{i}}+\left\|\dot{h}_{j}(t)\right\|_{H^{k_{j}(\Omega)}}^{p_{j} / q_{i}}\right)\right. \\
& \left.\left.+\left\|\dot{x}_{i}\right\|_{H^{k_{i}(\Omega)}}^{p_{i}-1}\right)\left\|\dot{h}_{i}(t)\right\|_{H^{k_{i}(\Omega)}}\right\} .
\end{aligned}
$$

We show that the right hand side of the above inequality is integrable. Indeed, for every $i$ a function

$$
\begin{aligned}
t \rightarrow & \left(b(t)+\sum_{j=1}^{n} 2^{p_{j-1}}\left\|\dot{x}_{j}(t)\right\|_{H^{k_{j}(\Omega)}}^{p_{j}}\left\|h_{j}(t)\right\|_{H^{k_{j}}(\Omega)}\right. \\
& \left.+\left\|\dot{h}_{j}(t)\right\|_{H^{k_{j}(\Omega)}}^{p_{j}}\left\|h_{i}(t)\right\|_{H^{k_{i}(\Omega)}}\right)
\end{aligned}
$$

is in $L^{1}[0, T ; \mathbb{R}]$, which follows by the estimation $\left\|h_{i}(t)\right\|_{H^{k_{i}+1}(\Omega)} \leq\left\|h_{i}\right\|_{\infty} \leq$ $\left\|h_{i}\right\|_{W^{1, p_{i}}}^{2}=c_{i}<\infty$. The integrability of a function

$$
\begin{aligned}
t \rightarrow c_{i}(t)+ & \left(\sum_{j \neq i}^{n} 2^{p_{j} / q_{i}-1}\left(\left\|\dot{x}_{j}(t)\right\|_{H^{k_{j}}(\Omega)}^{p_{j} / q_{i}}+\left\|\dot{h}_{j}(t)\right\|_{H^{k_{j}(\Omega)}}^{p_{j} / q_{i}}\right)\right. \\
& \left.+\left\|\dot{x}_{i}(t)\right\|_{H^{k_{i}(\Omega)}}^{p_{i}-1}\right)\left\|\dot{h}_{i}(t)\right\|_{H^{k_{i}(\Omega)}}
\end{aligned}
$$

follows by Hölder's inequality for every $i$.

In consequence Leibniz formula of differentiation under integral sign applies. By (3.2), (3.3) we obtain for $i=1,2, \ldots, n$

$$
\begin{aligned}
& \nabla_{x_{i}} g(\cdot, x(\cdot), \dot{x}(\cdot)) \in L^{1}\left[0, T ; H^{k_{i}}(\Omega)\right], \\
& \nabla_{\dot{x}_{i}} g(\cdot, x(\cdot), \dot{x}(\cdot)) \in L^{q_{i}}\left[0, T ; H^{k_{i}}(\Omega)\right]
\end{aligned}
$$

We also have

$$
\begin{aligned}
& \sum_{i=1}^{n} \int_{0}^{T}\left(\left\langle\nabla_{x_{i}} g(t, x(t), \dot{x}(t)), h_{i}(t)\right\rangle_{H^{k_{i}}(\Omega)}\right. \\
& \left.+\left\langle\nabla_{\dot{x}_{i}} g(t, x(t), \dot{x}(t)), \dot{h}_{i}(t)\right\rangle_{H^{k_{i}(\Omega)}}\right) d t \\
& \leq \sum_{i=1}^{n} c_{i}\left\|h_{i}\right\|_{\infty}+\sum_{i=1}^{n} d_{i}\left\|\dot{h}_{i}\right\|_{L^{p_{i}}\left[0, T ; H^{k_{i}}(\Omega)\right]} \\
& \leq \sum_{i=1}^{n}\left(c_{i}+d_{i}\right)\left\|h_{i}\right\|_{W^{1, p_{i}}\left[0, T ; H^{k_{i}(\Omega)}\right]} .
\end{aligned}
$$

Hence a directional derivative in the direction $h$ is given by the formula (3.4). 
It remains to show that the mapping $F^{\prime}: W \rightarrow(W)^{*}$ is continuous. It suffice to demonstrate that mappings

$$
\begin{aligned}
& W \ni x \mapsto \nabla_{x_{i}} g(\cdot, x(\cdot), \dot{x}(\cdot)) \in L^{1}\left[0, T ; H^{k_{i}}(\Omega)\right], \\
& W \ni x \mapsto \nabla_{\dot{x}_{i}} g(\cdot, x(\cdot), \dot{x}(\cdot)) \in L^{q_{i}}\left[0, T ; H^{k_{i}}(\Omega)\right]
\end{aligned}
$$

are continuous for $i=1,2, \ldots, n$. We use Theorem 2.2. Let us observe that strong convergence of $x_{n}$ in $W^{1, k_{i}}$ means strong convergence in $C[0, T$;

$\left.H^{k_{i}+1}(\Omega)\right]$ and that sequences $\dot{x}_{n}$ are strongly convergent in $L^{p_{i}}\left[0, T ; H^{k_{i}}(\Omega)\right]$ for $i=1,2, \ldots, n$. Put

$$
\begin{aligned}
& L_{1}=L^{p_{1}}\left[0, T ; H^{k_{1}}(\Omega)\right], L_{2}=C\left[0, T ; H^{k_{1}+1}(\Omega)\right], \ldots, \\
& L_{2 n-1}=L^{p_{n}}\left[0, T ; H^{k_{n}+1}(\Omega)\right], L_{2 n}=C\left[0, T ; H^{k_{n}}(\Omega)\right]
\end{aligned}
$$

and $L=L_{1} \times \ldots \times L_{2 n}$. By (3.2), (3.3), Lemma 2.1 we obtain by Theorem 2.2 that mappings (3.5) and (3.6) are continuous.

\section{References}

[1] Ambrosetti, A., Prodi, G., A Primer of Nonlinear Analysis, Cambridge Studies in Advanced Mathematics 34, Cambridge University Press, Cambridge, 1993.

[2] Appell, J., Upper estimates for superposition operators and some applications, Ann. Acad. Sci. Fenn. Ser. A I Math. Dissertationes 8(1) (1983), 149-159.

[3] Barbu V., Precupanu, Th., Convexity and Optimization in Banach Spaces, Mathematics and its Applications 10, D. Reidel Publ. Comp., Dordrecht, 1986.

[4] Brezis, H., Analyse fonctionelle: théorie et applications, Masson, Paris, 1983.

[5] Dinca, G., Pasca, D., Existence theorem of periodical solutions of Hamiltonian systems in infinite-dimensional Hilbert spaces, Differential Integral Equations 14(4) (2001), 405-426.

[6] Evans, L. C., Partial Differential Equations, Graduate Studies in Mathematics 19, Amer. Math. Soc., Providence, RI, 1998.

[7] Hille, E., Philips, R. S., Functional Analysis and Semi-groups, Amer. Math. Soc. Colloquium Publications 31, Providence, RI, 1957.

[8] Idczak, D., Rogowski, A., On the Krasnosel'skii Theorem - a short proof and some generalization, J. Austral. Math. Soc. Ser. B, (to appear).

[9] Krasnosel'skii, M. A., Topological Methods in the Theory of Nonlinear Integral Equations, A Pergamon Press Book The Macmillan Co., New York, 1964.

[10] Lieb, E. H., Loss, M., Analysis, Graduate Studies in Mathematics 14, Amer. Math. Soc., Providence, RI, 1997.

[11] Mawhin, J., Metody wariacyjne dla nieliniowych problemów Dirichleta (in Polish), WNT, Warszawa, 1994.

[12] Mawhin, J., Willem, M., Critical Point Theory, Springer-Verlag, New York, 1989.

[13] Nowakowski, A., Rogowski, A., On the new variational principles and duality for periodic solutions of Lagrange equations with superlinear nonlinearities, J. Math. Anal. Appl. 264 (2001), 168-181.

[14] Yosida, K., Functional Analysis, Springer-Verlag, New York, 1974. 
ON A CERTAIN GENERALIZATION OF THE KRASNOSEL'SKII THEOREM 147

Marek GaLewski

FACUlTy OF MATHEMATICS

UNIVERSITY OF ŁÓDŹ

BANACHA 22

90-238 ŁÓDŹ

POLAND 